\title{
The Subaru Coronographic Extreme AO (SCExAO) system: implementation and performances of the Coronographic Low Order WaveFront Sensor
}

Vogt, Frédéric, Martinache, Frantz, Guyon, Olivier, Yoshikawa, Takashi, Yokochi, Kaito, et al.

Frédéric Vogt, Frantz Martinache, Olivier Guyon, Takashi Yoshikawa, Kaito Yokochi, Vincent Garrel, Taro Matsuo, "The Subaru Coronographic Extreme AO (SCExAO) system: implementation and performances of the Coronographic Low Order WaveFront Sensor," Proc. SPIE 7736, Adaptive Optics Systems II, 773612 (28 July 2010); doi: 10.1117/12.857915

EviE Event: SPIE Astronomical Telescopes + Instrumentation, 2010, San Diego, California, United States 


\title{
The Subaru Coronographic Extreme AO (SCExAO) system : implementation and performances of the Coronographic Low Order WaveFront Sensor
}

\author{
Frédéric Vogt ${ }^{a, b}$, Frantz Martinache ${ }^{a}$, Olivier Guyon ${ }^{a}$, Takashi Yoshikawa ${ }^{a, c}$, Kaito Yokochi $^{a, d}$, \\ Vincent Garrel $^{a}$ and Taro Matsuo ${ }^{a}$ \\ ${ }^{a}$ National Astronomical Observatory of Japan, Subaru Telescope, Hilo, HI 96720, USA \\ ${ }^{b}$ Laboratoire d'Astrophysique, Ecole Polytechnique Fédérale de Lausanne, Observatoire de \\ Sauvergny, 1290 Versoix, Switzerland \\ ${ }^{c}$ Faculty of Engineering, The University of Tokyo, 7-3-1 Hongo, Bunkyo-ku, Tokyo 113-8656, \\ Japan \\ ${ }^{d}$ Graduate School of Engineering, Tokyo University of Agriculture and Technology, 2-24-16 \\ Naka-cho, Koganei, Tokyo 184-8588, Japan
}

\begin{abstract}
The Subaru Coronagraphic Extreme AO project (SCExAO) is a high performance coronagraph designed to deliver high contrast at small angular separation. For the detection of structures near the diffraction limit, an accurate control of low order wavefront aberrations - tip-tilt and focus - is essential as these aberrations create light leaks that are the source of confusion in the final science image. To address this major difficulty, we have equipped SCExAO with a specially designed Coronagraphic Low Order WaveFront Sensor (CLOWFS) using defocused images of a reflective ring located in the focal plane, that can track tip-tilt errors as small as $10^{-3} \lambda / D$. CLOWFS was originally designed to drive actuators in a closed-loop. Here, we show that it can also be used in post-processing to efficiently subtract the tip-tilt induced coronagraphic leaks in the final science image.
\end{abstract}

Keywords: Extrasolar Planets, Adaptive Optics, Coronography, CLOWFS, PIAA, SCExAO, Subaru telescope

\section{INTRODUCTION}

At near infrared wavelengths, an 8-meter class telescope such as Subaru provides sufficient angular resolution (40 milli-arcseconds at $\lambda \cong 1.6 \mu \mathrm{m})$ to be able to detect companions in the Habitable Zone of nearby stars $(\mathrm{d}<30$ pc). High contrast imaging near the diffraction limit however requires very good control and calibration of the wavefront aberrations in the optical system. From the ground, this task is complicated by the rapidly changing atmospheric wavefront creating speckled images. While Adaptive Optics (AO) offers a major improvement, performance is still limited and the direct imaging of planets often requires post-processing techniques such as Angular Differential Imaging (ADI)[1], which is most efficient at large $(\geq 10 \lambda / D)$ angular separations.

Imaging of companions close to the edge of the occulting mask in a coronagraph is very sensitive to low order aberration modes such as pointing and focus. Of these aberrations, pointing is especially critical since a tip-tilt excursion along a given direction will exactly mimic the signal of a true companion at a small angular separation in a coronographic image. To address this issue, Guyon et al. ${ }^{2}$ have proposed the Coronagraphic Low Order WaveFront Sensor (CLOWFS), a scheme which uses the light occulted by the focal plane mask as a an accurate pointing tracker.

The idea of using the light otherwise lost in the coronagraphic focal plane for tracking is not new and was for instance successfully implemented on the LYOT project[3]. The originality of CLOWFS resides in its dual-zone focal plane mask, designed to suppress a strong offset to the signal, carrying most of the power but no information, in a manner reminiscent of strioscopy. The suppression of this offset turns the otherwise imperceptible changes

Send correspondence to frantz@naoj.org

Adaptive Optics Systems II, edited by Brent L. Ellerbroek, Michael Hart, Norbert Hubin, Peter L. Wizinowich, Proc. of SPIE Vol. 7736, 773612 - (C) 2010 SPIE · CCC code: 0277-786X/10/\$18 - doi: 10.1117/12.857915 
due to small pointing errors into macroscopic changes of the CLOWFS image. Using this scheme, Guyon et al. ${ }^{2}$ were able to stabilize tip-tilt at the level of $10^{-3} \lambda / D$ in a closed-loop system at $633 \mathrm{~nm}$.

The same technique can be used to post-process science data and achieve an efficient substraction of coronographic leaks due to tip-tilt residuals in a long exposure. Using the SCExAO system[4] as a testbed, Vogt et al. ${ }^{5}$ experimentally demonstrate $\sim 2$ orders of magnitude of improvement of the detection limit over classical calibration procedures at angular separation of a few $\lambda / D$. In this paper, we will present the implementation and performance of the CLOWFS on the Subaru Coronographic Extreme AO (SCExAO) system (cf. Martinache et al, this conference) and its performances in laboratory.

This paper is organized as follows : in Section 2, we introduce how to use CLOWFS for post processing of coronographic images. In Section 3, we describe the implementation of the concept on the SCExAO experiment of the Subaru telescope, and we present our results in Section 4. Section 5 summarizes our results and discusses possible updates for the current CLOWFS configuration.

\section{PRINCIPLE}

\subsection{CLOWFS architecture}

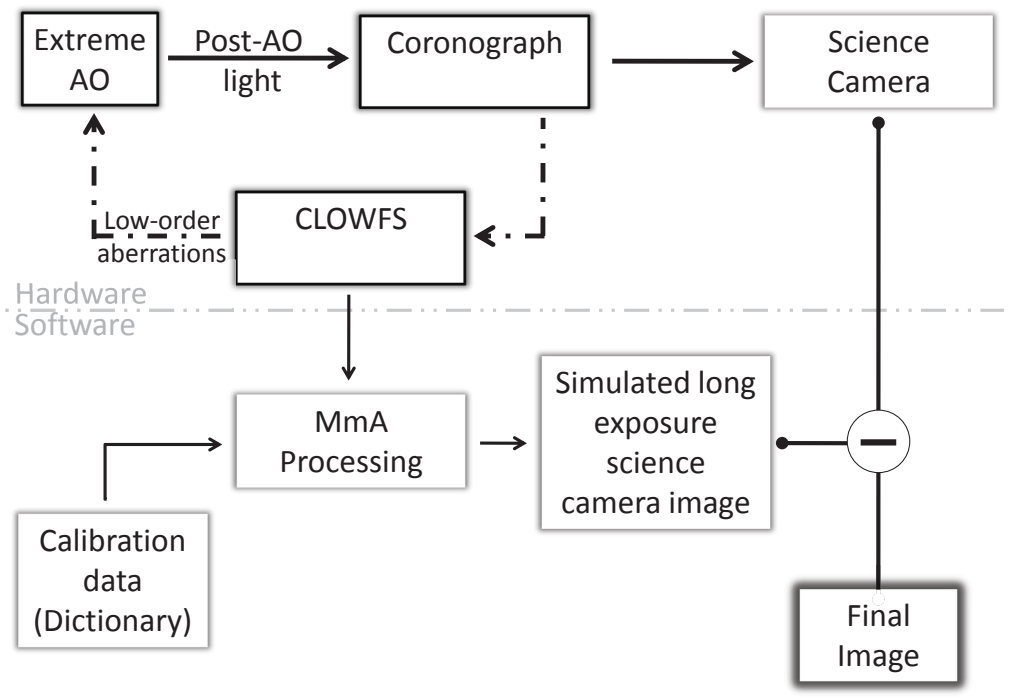

Figure 1. CLOWFS hard- and software global architecture.

Fig. 1 illustrates the implementation of the suggested post-processing technique of the coronagraphic images using CLOWFS. The hardware requirements are identical to the original design by Guyon et al. ${ }^{2}$ In addition to using the CLOWFS pointing error measurements in a real-time, closed-loop, at a frequency limited by the actuators, the CLOWFS signal can be used to predict the contribution to the light recorded on the science (i.e. post-coronagraph) camera. Only, rather than relying on a necessarily imperfect model of the optical train, we propose a more pragmatic approach.

During the (long) exposure on the science camera, the CLOWFS camera acquires a continuous stream of short (typically millisecond) exposures that record the instantaneous pointing error in both directions. Guyon et al. ${ }^{2}$ have demonstrated that over a small range $(\sim 0.2 \lambda / D)$ of pointing errors, a linear model relates the changes in CLOWFS images to the actual pointing errors, and took advantage of this in a closed-loop system, stabilizing the pointing at the level of $10^{-3} \lambda / D$ over extended periods of time $(\sim 1 \mathrm{hr})$. This is symbolised by the clockwise loop in a dashed-line format in Fig. 1. Note that the post-processing technique is not-restricted to the closed-loop case and can very well handle a wide range of pointing errors: the results presented in this work were obtained in this open-loop scenario. 
The key concept behind CLOWFS is the one-to-one correspondence that exists between simultaneous images acquired on the science and the CLOWFS camera. Keeping track of the CLOWFS images during a long exposure on the science camera is sufficient to determine the fraction of the light contained in the coronagraphic image, that can be attributed to pointing errors. This can then be subtracted from the science image, leading to a final tip-tilt residuals calibrated image, therefore improving contrast limits.

In its simplest version (see Section 5 for a discussion of the possible complements), the post-acquisition calibration of pointing errors with CLOWFS is a three-step procedure, summarized in Fig. 2.

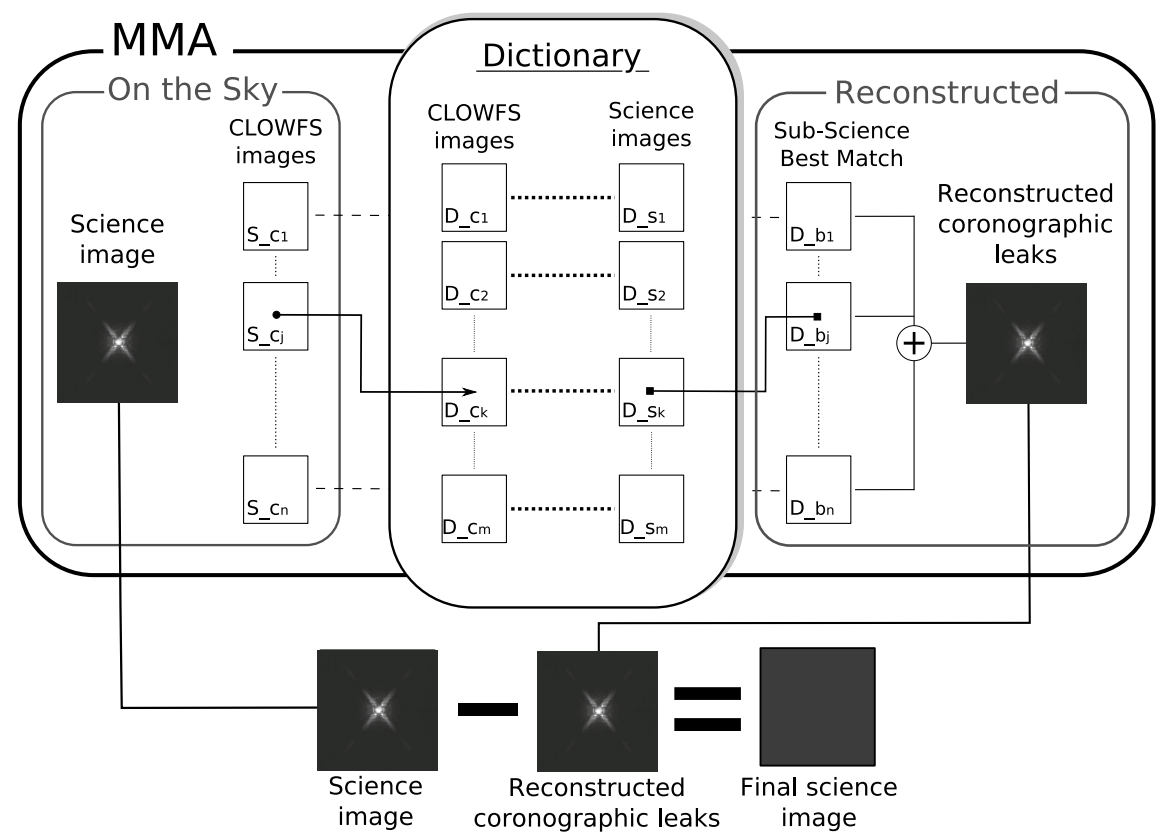

Figure 2. CLOWFS post-acquisition pointing errors calibration procedure, defined as the Match-Maker Algorithm (MMA).

- Step 1: characterize the configuration of the system and its response to low-order aberrations, using simultaneous images taken on both cameras (CLOWFS and coronagraphic). We will refer to this as the dictionary construction (see Section 2.2 below).

- Step 2 : during an exposure on a source of interest (a.k.a. science-target) with the coronagraphic camera, continuously acquire images at fast frame rate with the CLOWFS camera (see Section 2.3 below).

- Step 3 : send the recorded CLOWFS images to the Match-Maker Algorithm (MMA) to process the Science image (see Section 2.3 below).

We will describe those steps in more details in the next two Sections.

\subsection{System calibration: building up the dictionary}

During step 1, pairs of simulatenous short exposure images are taken with both the CLOWFS (labelled $\mathrm{D}_{c_{k}}$ ) and science $\left(\mathrm{D}_{s_{k}}\right)$ camera. They are stored in a database, forming what will be refered to as the dictionary. The pairs in the dictionary are used to decode the CLOWFS images $\left(\mathrm{S}_{c_{k}}\right)$ obtained during the observation into a corresponding coronographic leak in the science image. The CLOWFS images therefore serves as the identification key to build the long exposure estimate of coronographic leaks in the science image. This tiptilt calibration procedure relies on one-to-one correspondence between simultaneous images on the science and CLOWFS cameras. Some non-common path errors can occur after the coronograph, but they can be kept small by minimizing the number of optics used after the CLOWFS focal-plane mask. 
A preliminary version of the dictionary can be compiled in the lab, using a calibration source. However to minimize systematic error terms due to slowly varying non-common path errors, it is important to complete it with more up-to-date images acquired on a series of calibration (non-resolved) stars of spectral type and magnitude comparable to the science target, so as to get the best possible match. Ideally, during acquisition on the calibration star, one wants to cover a range of pointing errors that is larger than during the science exposure. For the laboratory results reported in this paper, we introduced a source of vibration to cover a wide range of pointing errors.

It is important to emphasize here that we do not explicitly calculate the tip-tilt errors, but rather directly observe the consequences of those errors via the CLOWFS system. And indeed, not having to calculate the tip-tilt errors has the huge advantage that, aside of being much simpler, no model of the coronograph is required, which would de facto require approximations of some sort. Ultimately, the ability to precisely characterize the coronographic leaks is determined by the size of the dictionary, which can be made aribitrarily large.

\subsection{Computing and subtracting coronagraphic leaks}

During a long (i.e. typically greater than one second) exposure on the science camera, the CLOWFS camera acquires a series of short exposures (Step 2), labelled $\mathrm{S}_{c_{k}}$ in Fig. 2. In post processing, a Match-Maker Algorithm (MMA) identifies, for each on-Sky CLOWFS image, the dictionary's CLOWFS image that provides the best match. The best match criterion is the Euclidian distance between the current CLOWFS and the dictionary images, which we define as follow :

$$
\sigma\left(S_{c_{k}}, D_{c_{k}}\right)=\sqrt{<\left(S_{c_{k}}-D_{c_{k}}\right)^{2}>}
$$

The operation is repeated for all images of the CLOWFS sequence. The short exposure science camera images selected $\left(\mathrm{D}_{b_{k}}\right)$ are co-added to form an estimate of the residuals in the long exposure coronagraphic image that can be attributed to pointing errors during the exposure on the science target. This final image is simply substracted from the long exposure to calibrate these pointing error residuals.

\section{LABORATORY DEMONSTRATION}

The experimental demonstration of the proposed post-processing technique was performed using the Subaru Coronagraphic Extreme-AO (SCExAO) bench in the Subaru laboratory [4]. Among extreme-AO systems, SCExAO specializes in the high-contrast characterization of the innermost $(<0.2$ arc sec.) surrounding of stars, and in that scope, implements a high-performance PIAA-based [6] coronagraph that takes full benefit of the angular resolution of the 8-meter Subaru Telescope. For our test, the special optics described by Lozi et al. ${ }^{7}$ were taken out of the beam and SCExAO was reduced to a conventional near-IR Lyot-coronagraph without a Lyot-stop [8]. SCExAO is operated at IR wavelength (H-band), and uses two identical uncooled fast readout InGaAs cameras (Xenics model XS-1.7-320), referred to as the science and CLOWFS camera in this paper.

Fig. 3 offers a schematic representation of the optical layout of SCExAO as used for our experiment. The light source is a $\lambda=1.55 \mu \mathrm{m}$ laser diode, illuminating a $17-\mathrm{mm}$ pupil mask emulating the Subaru Telescope pupil. The focal-plane mask is $85 \mu \mathrm{m}$ in radius, which given the f-number of the beam gives a $4.75 \lambda / D$ inner working angle (IWA). Note that when the PIAA is inserted in the beam, the focal plane scale changes [9] and the IWA becomes $1.5 \lambda / D$.

As described by Guyon et al., ${ }^{2}$ the CLOWFS focal-plane mask is made up of two zones: absorbing within a central disk and reflective in the adjacent annular region. The radius of the inner non-reflective region is $40 \%$ of the total radius. $\mathrm{A} \sim 20^{\circ}$-tilt of the mask with respect to the inital beam is sufficient to prevent beam collision and does not require an elongated (elliptical) mask. The light reflected by the mask is then re-imaged on the CLOWFS detector, slightly out of focus as suggested by Guyon et al., ${ }^{2}$ to solve the sign uncertainty in the determination of focus errors. Fig. 4 shows one example of a typical CLOWFS image.

For this experiment, a total of 15000 pairs of 1 millisecond exposure images were acquired with the CLOWFS and coronagraphic cameras on the SCExAO testbed at a $\sim 10 \mathrm{~Hz}$ frame rate. A total of 14850 images are used to build the dictionary (images $\mathrm{D}_{c_{k}}$ and $\mathrm{D}_{s_{k}}$ ). A serie of 150 contiguous pairs of images in the middle 


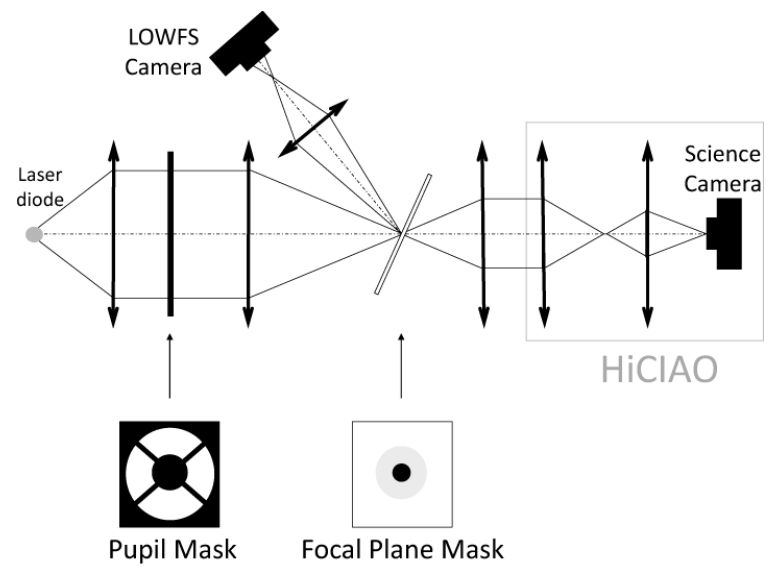

Figure 3. Optical layout of SCExAO used in Lyot-coronagraph mode. A mask lit by an IR $(\lambda=1.55 \mu m)$ laser diode emulates the Subaru Telescope pupil. The beam is focused on the dual-zone focal-plane occulting mask described in the text. The light reflected by the focal-plane mask feeds the (slightly defocused) CLOWFS camera, used to characterize pointing errors. The light that is not intercepted by the mask is then re-imaged on the science camera. Examples of images obtained on both cameras are presented in Fig. 4 and 5.

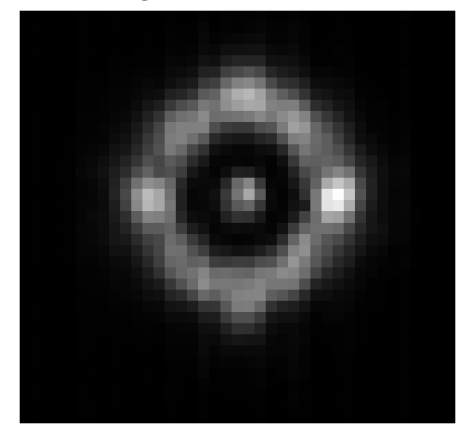

Figure 4. Example of a CLOWFS image. Variations of the repartition of intensity in the image of the reflective annular region of the mask can be used to trace pointing errors.

of this sequence were used to simulate a single observation : the coronagraphic images were dark substracted and coadded to form our simulated coronagraphic image, while the sequence of CLOWFS images was simply stored (they form the $\mathrm{S}_{c_{k}}$ list of images). We then applied the MMA as described previously. The result is shown in Fig. 5. The raw 150s coadded Science frame is shown in (panel a) and the result of the proposed calibration and post-processing procedure is shown in(panel c). In (panel b), we display the resulting image when doing a standard PSF substraction. To simualte such a PSF, we randomly select $150 \mathrm{D}_{s_{k}}$ Science images in the dictionary, and co-add them to create a random PSF reconstructed image, that we substract from the raw Science image.

\section{RESULTS}

\subsection{Understanding CLOWFS images}

As we mentioned previously, a CLOWFS image contains information on the amount and type of coronographic leaks present in the system. Let us consider two CLOWFS images $I_{1}, I_{2}$. The Euclidian distance $\sigma\left(I_{1}, I_{2}\right)$, defined in Eqt. 1, can be seen as an indicator of the similarity or match in between the two images. A perfect match would for example have $\sigma\left(I_{1}, I_{2}\right)=0$. The Euclidian parameter is a key parameter for the MMA procedure as described previously. It can also be used to study the content of the dictionary, which is done by Vogt et al. ${ }^{5}$ In Section 4.2, we describe further the obtained results for the Science image, and compare the efficiency of the MMA procedure as compared to the standard PSF substraction method.

It should be mentioned here that the CLOWFS images capture and code more than tip-tilt errors. However, we do not adress here those other, higher-order, wavefront errors (such as focus for example). We concentrate 

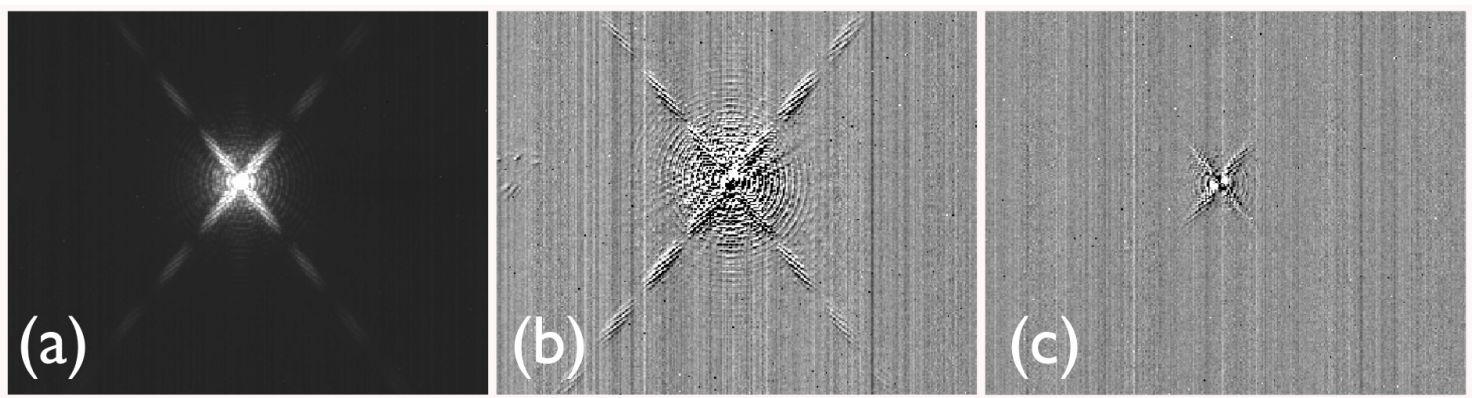

Figure 5. a) Long-exposure "science camera" image, non-linear intensity scale. Airy rings and diffraction spikes due to the peculiar Subaru Telescope pupil are clearly visible. b) Image after standard substraction of a PSF without using the proposed CLOWFS image selection procedure. c) Image after CLOWFS post-processing calibration. Panels b and c use the same intensity scale.

solely on tipt-tilt excursion so as to demonstrate the concept of the CLOWFS used for post-processing. But clearly, those higher-order wavefront can be handled by the CLOWFS system in a similar way, a point which will be implemented when using the proposed system on real science targets.

\subsection{The MMA efficiency}

Fig. 5 shows three images that demonstrate the power of the proposed approach, while Fig. 6 plots the associated radial profiles. The raw data (panel a in Fig. 5, dashed-line in Fig. 6) corresponds to a simulated long exposure, acquired after the coronagraph, without a Lyot-stop. If this was an actual science target possibly hosting a companion, a fairly standard calibration method of tip-tilt residuals would be to subtract from this an image acquired under identical conditions on a calibration star. This is simulated here by picking a random set of images from the complete sequence of science images in the dictionary. The example of one such subtraction is represented in the panel b of Fig. 5. A series of 200 different radial profiles labeled Random match in Fig. 6 show that this approach in average improves the detectability by a factor 10 . This baseline calibration method can serve as a comparison with the CLOWFS tip-tilt calibration method we propose: panel c in Fig. 5 shows the result of this calibration: features like the diffraction spikes visible in panels a and $\mathrm{b}$ fall completely under the readout noise of the detector. Fig. 6 (solid line, labeled best match) quantifies the associated gain in sensitivity, which is improved by almost two orders of magnitude at $4.75 \lambda / D$. Note that normally, SCExAO uses the PIAA that changes the focal plane scale, which brings the inner working angle at $1.5 \lambda / D$.

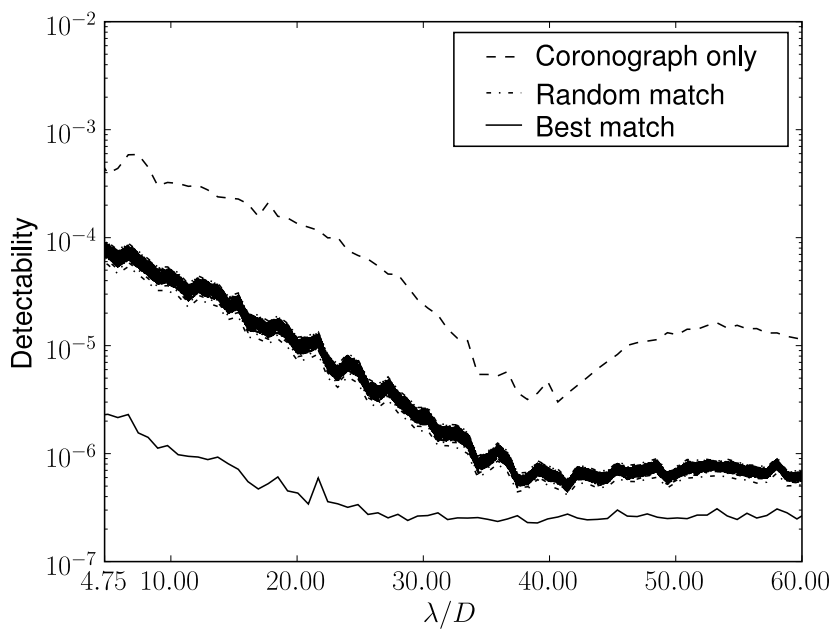

Figure 6. Radial detectablity of the raw Science image (dot-dashed line), the best-fit cleaned Science image (full line) and 200 random standard PSF substracted images. See in the text for details. 


\section{DISCUSSION}

The results presented in Section 4. demonstrate that, by calibrating pointing errors in post-processing, CLOWFS improves the sensitivity of a coronagraph by a factor near $10^{2}$. Given its simplicity of implementation, CLOWFS appears like a very attractive concept that SCExAO will employ for its commissioning run.

One critical advantage of a CLOWFS and the MMA suggested is that no modelling of the coronograph is required, and litterally no assumptions on the system need to be made. Indeed, dealing directly with the consequences of coronographic leaks, and using those consequences to characterize and correct the leaks themselves makes this technique extremely robust, and able to handle many different situations and types of coronographic leaks.

The rudimentary approach proposed here can certainly be improved. The construction of a bigger dictionary, built over a long period of time, and over many stars, would expand the level of description of the instrument. And in turn better describe and decode the coronographic leaks. Extended knowledge of several experimental parameters, such as the telescope elevation, star color or magnitude, could be implemented in the dictionary to help decoding faster and better the recorded CLOWFS images.

A big dictionary, covering large tip-tilt excursions, while improving the matching of CLOWFS on-Sky images, might slow down the MMA in this basic setup. In this big dictionary, CLOWFS images for small tip-tilt excursions will look extremely similar. And thus, a possible update to the MMA would be to remove some of those similar images and implement a Principal Components Analysis for small tip-tilt excursions. This can be expected to work well, as CLOWFS images are a linear function of aberrations in the small aberrations regime, a fact which is true for ExAO.

The CLOWFS and MMA post-processing algorithm described in this paper are being implemented on the SCExAO experiment on the Subaru telescope. The matching algorithm will be improved so as to be able to handle coronagraphic leaks due to higher order wavefront aberrations. Because it makes no assumption on targets and the current state of the optical bench, CLOWFS used both for live and post-processing is likely to play a significant part to the success of SCExAO, that specializes in the detection of planetary companions at small angular separation.

\section{Acknowledgments}

Vogt thanks Brian.

\section{REFERENCES}

[1] Marois, C., Lafrenière, D., Doyon, R., Macintosh, B., and Nadeau, D., "Angular Differential Imaging: A Powerful High-Contrast Imaging Technique," ApJ 641, 556-564 (Apr. 2006).

[2] Guyon, O., Matsuo, T., and Angel, R., "Coronagraphic Low-Order Wave-Front Sensor: Principle and Application to a Phase-Induced Amplitude Coronagraph," ApJ 693, 75-84 (Mar. 2009).

[3] Digby, A. P., Hinkley, S., Oppenheimer, B. R., Sivaramakrishnan, A., Lloyd, J. P., Perrin, M. D., Roberts, Jr., L. C., Soummer, R., Brenner, D., Makidon, R. B., Shara, M., Kuhn, J., Graham, J., Kalas, P., and Newburgh, L., "The Challenges of Coronagraphic Astrometry," ApJ 650, 484-496 (Oct. 2006).

[4] Martinache, F. and Guyon, O., "The Subaru Coronagraphic Extreme-AO Project," in [Society of PhotoOptical Instrumentation Engineers (SPIE) Conference Series], Presented at the Society of Photo-Optical Instrumentation Engineers (SPIE) Conference $\mathbf{7 4 4 0}$ (Aug. 2009).

[5] Vogt, F., Martinache, F., Guyon, O., Takashi, Y., Kaito, Y., Vincent, G., and Taro, M. (2010). In preparation.

[6] Guyon, O., "Phase-induced amplitude apodization of telescope pupils for extrasolar terrestrial planet imaging," A\&BA 404, 379-387 (June 2003).

[7] Lozi, J., Martinache, F., and Guyon, O., "Phase-Induced Amplitude Apodization on Centrally Obscured Pupils: Design and First Laboratory Demonstration for the Subaru Telescope Pupil," PASP 121, 1232-1244 (Nov. 2009).

[8] Aime, C. and Soummer, R., "Introduction to stellar coronagraphy with entrance pupil apodization," in [EAS Publications Series], C. Aime \& R. Soummer, ed., EAS Publications Series 8, 79-92 (2003). 
[9] Guyon, O., Pluzhnik, E. A., Galicher, R., Martinache, F., Ridgway, S. T., and Woodruff, R. A., "Exoplanet Imaging with a Phase-induced Amplitude Apodization Coronagraph. I. Principle," ApJ 622, 744-758 (Mar. 2005). 